\title{
Base Qualis e a indução do uso de periódicos da área de Psicologia'
}

\section{Qualis Base and the induction of use of journals in Psychology area}

Maria do Carmo Moreira JACON²

\section{RES U MO}

A presente pesquisa, de natureza exploratória, visa verificar se a base Qualis, uma vez implementada, induziu o uso dos periódicos nela melhor classificados, especialmente periódicos de conceito A, de abrangência nacional. Para tal objetivo, definiu-se como objeto de estudo periódicos Qualis da área de Psicologia e, como contexto, o Programa de Pós-Graduação em Psicologia da Pontifícia Universidade Católica de Campinas. $\bigcirc$ universo pesquisado constou de uma amostra de teses e dissertações defendidas no período 1997-2002 e de uma população de mestrandos e doutorandos matriculados no referido Programa. Os procedimentos metodológicos empregados foram as técnicas de análise de citação e de aplicação de questionário. A análise dos resultados revelou que a atribuição de conceito máximo não induz necessariamente ao uso efetivo. Conclui-se, a partir desse fato, que o consenso (avaliação pelos pares) que fundamenta a base Qualis e que é empreendido na classificação de periódicos científicos da área de Psicologia, não se reflete no consenso dos segmentos (docentes e discentes) do Programa analisado.

Palavras-chave: periódico científico; pós-graduação; base Qualis; psicologia; Pontifícia Universidade Católica de Campinas.

\section{A B S T R A C T}

The present research, of exploratory nature, aims at verifying whether the Qualis base, once implemented, has led researchers to the use of journals classified therein, especially those with A concept, published nationwide. For that aim, we have defined as the object of study, the Qualis classified journals on Psychology, and, as our research context, the Graduate Program in Psychology (Programa de Pós-Graduação em Psicologia) of the Pontifícia Universidade Católica de Campinas, in the state of São Paulo, Brazil. The universe we have researched was composed of a sample of thesis and dissertations completed during the period of 1997-2002, besides a population of graduate students enrolled in the Program, candidates to the Master and Ph.D. degrees. The methodological procedures employed herein are the citation analysis and the questionnaire application. The analysis of results has shown that a journal's maximum classification awarded by Qualis, did not effectively lead to the use of such journal. Therefore, the conclusion is that the consensus (evaluation by their piers) upon which the Qualis base establishes its classification of scientific journals in Psychology, is not reflected on the consensus of the segments (professors and graduate students) at the analyzed Program.

Keywords: Scientific journals; graduate programs; Qualis base; psychology; Pontifícia Universidade Católica de Campinas.

\footnotetext{
1 Artigo elaborado a partir da dissertação de M.C.M. JACON, intitulada "Base Qualis: uso e qualidade de periódicos científicos no Programa de Pós-Graduação em Psicologia da Pontifícia Universidade Católica de Campinas", 2006. Orientação: Profa. Dra. Maria de Fátima G. M. Tálamo.

2 Mestre em Ciência da Informação. Responsável pelo Serviço de Publicação, Divulgação e Intercâmbio, Sistema de Bibliotecas e Informação, Pontifícia Universidade Católica de Campinas. Rua Marechal Deodoro, 1099, Centro, 13010-920, Campinas, SP, Brasil. E-mail: <mariadocarmo@puc-campinas.edu.br>.

Recebido em 17/7/2006 e aceito para publicação em 8/2/2007.
} 


\section{NTRODUÇÃ O}

A pesquisa no campo do conhecimento, para que lhe seja atribuído o valor científico, necessita do reconhecimento e aceitação pelos pares. Em síntese, sem sua literatura, uma área científica não poderá existir. E para que a pesquisa seja reconhecida e integrada ao "corpus" de conhecimento, precisa ser comunicada.

Garvey (1979, p. 10) reconhece o processo de comunicação científica como um conjunto de atividades associadas à produção, disseminação e uso da informação, desde o momento em que o cientista concebe uma idéia para pesquisar, até que a informação acerca dos resultados seja aceita como constituinte do conhecimento científico.

Em suma, para que a pesquisa tenha valor científico é necessário que seja avaliada e validada através da publicação em um canal formal de divulgação. Dentre os canais existentes, os periódicos científicos apresentam todas as características de que os pesquisadores necessitam para promover a circulação e uso de suas pesquisas: são editados em pequenos intervalos, seus artigos trazem os avanços da ciência e alcançam uma grande audiência, fato proporcionado pela indexação de artigos em bases de dados nacionais e internacionais.

A disseminação proporcionada pela indexação, por sua vez, faz com que os periódicos científicos desempenhem também, além da função de veículo de divulgação de pesquisas do autor/pesquisador, a função de fonte de informação para o usuário.

Além desses atributos, os periódicos onde os pesquisadores publicam seus artigos, têm ainda outro importante papel: o de ser um dos itens de maior peso na avaliação do sistema nacional de pós-graduação brasileiro.

A produção científica dos pesquisadores vinculados à pós-graduação é valorizada e indica a dedicação de docentes e discentes quanto ao desenvolvimento de pesquisas. Na avaliação dos programas, a quantidade das pesquisas publicadas em periódicos científicos contribui de forma significativa ao cômputo total dos pontos alcançados e da nota atribuída a cada programa.

A produção intelectual dos programas é sem dúvida fator decisivo na avaliação final. A experiência acumulada pela Coordenação de Aperfeiçoamento de
Pessoal de Nível Superior (CAPES) nas avaliações, demonstra que o "[...] desempenho dos programas possui estreita relação e coerência entre os quesitos, ou seja, um programa que possui uma boa produção intelectual dificilmente apresenta problemas um outros aspectos avaliados" (Coordenação..., 2004).

A avaliação de periódicos científicos é importante não só para subsidiar a avaliação dos programas, mas também para fornecer uma cartografia da produção científica brasileira, pois segundo Valério (1994 p.121), os periódicos científicos refletem a ciência produzida de um país e, portanto, é imperativo avaliar-se essa ciência. A importância de avaliar-se a produção científica publicada em periódicos científicos é reflexo, segundo Ziman (1979), da própria concepção de ciência: os fatos e teorias devem passar por análises críticas de outros pesquisadores (avaliação pelos pares) antes de serem aceitos universalmente.

\section{Avaliação de Periódicos Científicos Brasileiros}

A CAPES avalia os programas de pós-graduação no Brasil, através do atendimento a critérios estabelecidos. Conforme mencionado, a produção científica é caracterizada como um dos itens mais importantes no sistema de avaliação de um programa e, devido a tal importância, foi necessária a criação de um instrumento que sistematizasse o processo, fornecendo indicadores que subsidiassem o sistema de avaliação dos programas.

A implantação da base Qualis foi a resposta a essa necessidade. É uma base de classificação dos veículos utilizados pelos programas de pós-graduação para a divulgação da produção intelectual de docentes e discentes e se fundamenta nas informações fornecidas pelos programas.

A classificação dos títulos é feita por comissões, composta de consultores ad hoc e representantes de área da CAPES. A escolha do instrumento fica a critério de cada área, desde que haja correlação com a metodologia proposta pela CAPES (avaliação pelos pares). A área da saúde utiliza, por exemplo, o Journal of Citation Report (JCR); outras áreas estabelecem critérios editoriais próprios ou utilizam o Fator de Impacto (FI) estabelecido pelo Institute for Scientific Information (ISI). Já a área de Psicologia adota como metodologia 
- modelo de avaliação que contempla aspectos intrínsecos (conteúdo) e extrínsecos (forma) dos veículos.

Os aspectos extrínsecos são objetivamente mensuráveis, portanto de fácil aferição, o que não ocorre com os aspectos intrínsecos, cuja avaliação contempla a análise de informações compiladas na publicação, tais como: critérios de arbitragem dos textos, qualidade do corpo editorial e consultores e qualidade dos artigos (originalidade e identificação com a orientação temática da revista). Em síntese, não se avalia diretamente o conteúdo do artigo, mas infere-se "[...] sobre a sua qualidade a partir de uma classificação atribuída ao veículo que o divulga" (Souza; Paula, 2002, p. 11).

Outra questão a ser analisada é que, a cada avaliação, 70\% dos componentes das comissões são trocados, podendo ocorrer divergências quanto à classificação atribuída em avaliações anteriores. Tal fato advém da subjetividade presente em um processo de avaliação, uma vez que o julgamento implica em juízo de valores, envolvendo o conhecimento e visão de mundo que cada indivíduo possui sobre determinado assunto.

Além das questões da mudança dos integrantes das comissões e da alteração nas avaliações subseqüentes, verifica-se, também, a necessidade da ampliação da articulação entre as áreas para a aplicação de critérios similares na classificação, pois o processo aponta pontuações diferentes aos mesmos periódicos usados por diferentes áreas.

A despeito da necessidade de revisão dos critérios e indicadores adotados e de padronização de instrumentos, foi concretizado o objetivo da base Qualis: realizar um tratamento sistemático e qualitativo da produção dos programas de pós-graduação, visando aperfeiçoar os indicadores que subsidiam a avaliação desses programas. A classificação dos periódicos pode ser consultada através da Internet, no sítio da CAPES. ${ }^{3}$

\section{Objetivo}

objetivo principal da presente pesquisa foi verificar se a base Qualis, uma vez implementada, induziu pesquisadores da área de Psicologia ao uso dos periódicos melhor classificados dessa área, de conceito A e abrangência nacional segundo a Qualis, na produção de teses e dissertações para o Programa de Pós-Graduação em Psicologia da PUC-Campinas, durante o período 1997-2002.

$\bigcirc$ estudo justifica-se por contribuir com resultados, cuja confrontação com dados empíricos permitiu investigar se o consenso (avaliação pelos pares) que fundamenta a base Qualis e que é empreendido na classificação de periódicos científicos, reflete-se no consenso dos segmentos (docentes e discentes) do Programa analisado. Os resultados obtidos podem contribuir para o estabelecimento de padrões de qualidade na avaliação científica brasileira. Podem contribuir igualmente para uma reflexão sobre a metodologia utilizada para a classificação de periódicos que, por sua vez, serão utilizados como instrumento de avaliação da atividade científica dos programas de pós-graduação.

\section{MATERIALE MÉTODOS}

Trata-se de uma investigação empírica de natureza exploratória quali-quantitativa. A análise quantitativa busca a freqüência dos temas e fornece interpretação dos dados explicitados, enquanto a qualitativa fornece as categorias necessárias para análise dos elementos implícitos.

objeto empírico desta pesquisa é composto de 50 títulos de periódicos da área de Psicologia (publicados no Brasil), classificados em relação à qualidade e âmbito (Anexo 1). Os títulos avaliados foram citados por docentes nos relatórios dos Programas de Pós-Graduação em Psicologia, no triênio 1998-2000, em lista enviada a CAPES. A Tabela 1 apresenta a distribuição da classificação dos periódicos científicos da área de Psicologia (1998-2000).

$\bigcirc$ universo estudado distribui-se em:

A. Amostra de 26 títulos, 10\% do total de 256 teses/dissertações defendidas no Programa de Pós-Graduação em Psicologia da PUC-Campinas, no período 1997-2002;

B. População de 15 sujeitos (4 doutorandos e 11 mestrandos), 14,5\% do total de 103 alunos matriculados nos Cursos de Mestrado e Doutorado do Programa até dezembro de 2005.

\footnotetext{
${ }^{3}$ Coordenação de Aperfeiçoamento de pessoal de Nível Superior (CAPES) Qualis - Classificação de Periódicos, Jornais e Revistas. Disponível em: <http://qualis.capes.gov.br>.
} 
Tabela 1. Distribuição da classificação dos periódicos avaliados (1998-2000).

\begin{tabular}{|c|c|c|c|c|}
\hline \multirow{2}{*}{ Âmbito } & \multicolumn{3}{|c|}{ Conceito } & \multirow{2}{*}{ Total } \\
\hline & $A$ & $B$ & $C$ & \\
\hline Nacional & 15 & 8 & 1 & 24 \\
\hline Local & 7 & 10 & 9 & 26 \\
\hline Total & 22 & 18 & 10 & 50 \\
\hline
\end{tabular}

A seleção do universo A foi feita de forma aleatória e teve como base de amostragem uma lista obtida na Biblioteca do Centro de Ciências da Vida (CCV) do Sistema de Bibliotecas e Informação (SBI) da PUC-Campinas, que comporta, entre outros, o acervo do Curso de Psicologia. A população do universo B foi determinada pela freqüência dos alunos ao Programa, no período da aplicação do questionário.

Os dados quantitativos foram obtidos com a técnica de Análise de Citação, através do levantamento de 1512 referências do universo $A$ (993 dissertações e 519 teses), Para estudo comparativo de uso, efetuouse o levantamento de citações, não só de títulos de periódicos Qualis da área de Psicologia, mas também de outros tipos de publicações (livros, outros periódicos, teses, dissertações ou outro material utilizado).

Os dados qualitativos foram coletados através de questionário estruturado, com perguntas abertas e fechadas, aplicado em consulta individual e em grupo.

\section{RESULTADOSE ANÁLISE DOS DADOS}

Os resultados mais significativos obtidos com a análise de citações e aplicação do questionário são descritos a seguir.

\section{Análise das Citações}

O levantamento de citações nas referencias bibliográficas do universo $\mathrm{A}$ apontou o seguinte resultado: 5 teses e 14 dissertações citaram periódicos Qualis da área de Psicologia, enquanto que 1 tese e 6 dissertações não os citaram. $O$ resultado indica, em um primeiro momento o uso expressivo de periódicos Qualis por mestrandos e doutorandos (73\%).

O levantamento apontou a citação de 14 periódicos Qualis, ou seja 28\% do total (50). A Tabela 2 elenca a relação dos títulos citados.

Tabela 2. Periódicos Qualis citados.

\begin{tabular}{lc}
\hline Âmbito e Títulos & Conceito \\
\hline Nacional & $\mathrm{A}$ \\
Arquivos Brasileiros de Psicologia & $\mathrm{A}$ \\
Estudos de Psicologia (PUC-Campinas) & $\mathrm{A}$ \\
Psico PUCRS & $\mathrm{A}$ \\
Psicologia: Ciência e Profissão & $\mathrm{A}$ \\
Psicologia em Estudo & $\mathrm{A}$ \\
Psicologia: Reflexão e Crítica & $\mathrm{A}$ \\
Psicologia: Teoria e Pesquisa & $\mathrm{A}$ \\
Psicologia USP & $\mathrm{B}$ \\
Alethéia & $\mathrm{B}$ \\
Cadernos de Psicologia (UFMG) & $\mathrm{B}$ \\
Psicologia Escolar e Educacional & $\mathrm{B}$ \\
Revista Brasileira de Crescimento e Desenvolvimento Humano & $\mathrm{B}$ \\
Temas de Psicologia & $\mathrm{C}$ \\
Boletim de Psicologia & \\
\hline
\end{tabular}

TransInformação, Campinas, 19(2):189-197, maio/ago., 2007 
Uma análise proporcional indica que os periódicos Qualis B foram mais utilizados $(62,5)$ do que os $A(53,3)$. O exame da tabela 2 aponta a citação de 8 títulos Qualis A e 5 Qualis B. Supunha-se, inicialmente, a utilização maior de periódicos Nacional A, uma vez que a atribuição de conceito máximo indica a excelência de tais publicações, refletindo artigos de pesquisadores experientes. $O$ resultado obtido, no entanto sinaliza um uso regular desses periódicos.

Quanto ao uso geral de periódicos classificados como nacionais demonstra a importância de uma publicação com maior circulação ou distribuição. Vale ressaltar que ao classificar periódicos em categorias local, nacional e internacional, está se atribuindo a cada nível um estágio de qualidade superior ao outro. Assim, há uma busca maior, não só por autores como também pela comunidade acadêmica por publicações classificadas em níveis elevados pela visibilidade proporcionada e pressuposta qualidade.

No conjunto de títulos Qualis pesquisados, a publicação Estudos de Psicologia (PUC-Campinas) se destacou dos demais por ser o título mais citado. É um periódico da "casa" e provavelmente inclui parte da produção científica dos corpos docente e discente. Esse fato, entretanto não caracteriza endogenia, já que para receber conceito A supõe-se que o periódico preencheu os critérios de avaliação quanto à política editorial, que estabelece que o veículo contenha um percentual de 20\%, ao ano, de autores com vínculo institucional. O fato de o periódico Estudos de Psicologia (PUC-Campinas) ser o mais citado pode indicar que os artigos publicados contêm trabalhos afinados com as linhas de pesquisa do Programa

O levantamento dos tipos de publicações citadas apresentou, conforme Tabela 3, cinco categorias definidas pelo estudo.

O levantamento das citações do Universo A (teses/dissertações) apontou a citação de 18 periódicos Qualis (repete-se o título): 14 dissertações citaram 14 títulos, enquanto que 5 teses citam 4 . Dada a proporção entre as amostras, o uso desses periódicos por mestrandos e doutorandos é quase idêntico.

Como se disse anteriormente, o fato de 19 trabalhos (73\%), de uma amostra de 26, citarem periódicos Qualis indica um bom uso dessas publicações. Porém, quando se compara a citação de periódicos Qualis (1,2\% do total de publicações citadas), verifica-se um uso classificado como fraco. A Figura 1 ilustra o percentual das citações recebidas.

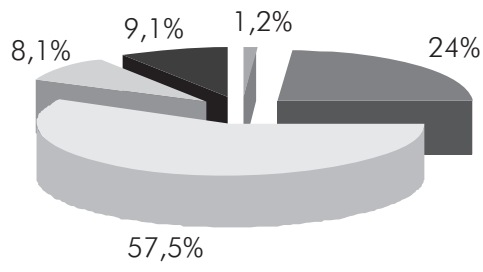

$\square$ Periódicos Qualis $\quad \square$ Teses/Dissertações/Monografias - Outros Periódicos घ Outros Tipos de Materiais $\square$ Livros

Figura 1. Percentual das citações recebidas.

Tabela 3. Tipos de publicações citadas e citações recebidas.

\begin{tabular}{lccc}
\hline \multirow{2}{*}{ Tipos de Publicações } & \multicolumn{2}{c}{ Citações Recebidas } & \multirow{2}{*}{ Total } \\
\cline { 2 - 4 } & Dissertações & Teses & 18 \\
\hline Periódicos Qualis & 14 & 4 & 363 \\
Outros Periódicos & 205 & 158 & 870 \\
Livros & 626 & 244 & 123 \\
Teses/Dissertações & 66 & 57 & 138 \\
Outros* & 82 & 56 & 1512 \\
\hline Total & 993 & 519 & 13 \\
\hline
\end{tabular}

* Outros refere-se a uma categoria maior que engloba anais, relatórios, palestras, trabalhos não publicados, projetos, jornais, revistas de divulgação, notas, textos on-line, ofício, filmes, músicas e vídeos. 
Os dados da Tabela 3 apontam, também, que "Outros Periódicos" não constituem a principal fonte de pesquisa do Programa estudado, contribuíram com $24 \%$ das citações. Já os livros obtiveram o maior número de citações nas teses e dissertações pesquisadas $(57,5 \%)$, isso porque, possivelmente, apresentam a base conceitual da Psicologia, onde se encontram obras clássicas de referência para a área, como as de Freud ou Yung.

A categoria "Outros" inclui outras publicações e recursos informacionais como filmes ou músicas. Importa ressaltar que contemplam número semelhante de citações de "Teses e Dissertações".

\section{Questionário}

Apresenta-se a seguir o resultado das questões mais pertinentes ao objetivo da presente pesquisa.

Uma das questões solicitou aos respondentes, em consulta individual e em grupo, que assinalassem o tipo de publicação mais utilizado nos trabalhos acadêmicos realizados, indicando apenas uma alternativa. Desconsideraram-se 2 respostas por inconsistência, pois foram assinaladas mais de uma alternativa. A Tabela 4 aponta os tipos de publicações utilizados.

Contrariando a tendência do uso de livros, apontada na análise das citações, os periódicos representam a categoria mais utilizada. Possivelmente são utilizados para pesquisa sobre tema e referências de trabalhos, mas não são citados ou então o uso passado não se repete no presente.

Outra questão solicitou a relação de 3 títulos (livros, periódicos ou outro tipo de publicação) que os entrevistados lembravam no momento. Nessa questão, houve a contribuição de 12 entrevistados. A tabela 5 apresenta a relação dos títulos de livros e periódicos mais lembrados.

Tabela 4. Tipos de publicação segundo utilização.

\begin{tabular}{|c|c|}
\hline Tipos de Publicações & Números Absolutos \\
\hline Livros & 4 \\
\hline Periódicos & 8 \\
\hline Teses/Dissertações & 1 \\
\hline Total & 13 \\
\hline
\end{tabular}

Tabela 5. Publicações segundo lembrança.

\begin{tabular}{|c|c|}
\hline \multirow{2}{*}{ Títulos } & Números \\
\hline & Absolutos \\
\hline \multicolumn{2}{|l|}{ Livros } \\
\hline Ser e fazer & 3 \\
\hline Yoga - Imortalidade liberdade & 1 \\
\hline O sagrado e o profano & 1 \\
\hline Muito além da deficiência & 1 \\
\hline Infância, educação e neoliberalismo & 1 \\
\hline Globalização & 1 \\
\hline Câncer como ponto de mutação & 1 \\
\hline Sobre o behaviorismo & 1 \\
\hline O lucro ou as pessoas & 1 \\
\hline Da pediatria à psicanálise & 1 \\
\hline Total & 12 \\
\hline \multicolumn{2}{|l|}{ Periódicos } \\
\hline Psicologia: Reflexão e Crítica & 4 \\
\hline Psicologia: Ciência e Profissão & 3 \\
\hline Psicologia USP & 2 \\
\hline Estudos de Psicologia (PUC-Campinas) & 2 \\
\hline Revista de Terapia Comportamental & 1 \\
\hline Estudos de Psicologia (Natal) & 1 \\
\hline Discorpo & 1 \\
\hline Motricidade & 1 \\
\hline ABRAPEE $^{\prime}$ & 1 \\
\hline Psicologia Escolar & 1 \\
\hline Psychê & 1 \\
\hline Cadernos de Psicologia & 1 \\
\hline Psicologia, Teoria e Pesquisa & 1 \\
\hline Revista de Psicologia & 1 \\
\hline $\mathrm{JABA}^{2}$ & 1 \\
\hline $\mathrm{JEAB}^{3}$ & 1 \\
\hline Behavior Analyst & 1 \\
\hline Total & 24 \\
\hline
\end{tabular}

A análise da Tabela 5 aponta dois aspectos:

- Parte dos títulos de livros lembrados não indica relação temática óbvia com a área de Psicologia;

- Os títulos de periódicos são mais lembrados e pertencem à área de Psicologia.

O fato de a maioria dos títulos de livros lembrados não ser explicitamente da área de Psicologia pode demonstrar interdisciplinaridade temática ou então 
que a lembrança momentânea é de um título qualquer, possivelmente lembrado porque constitui uma leitura marcante. Nesses casos, tais obras podem servir de estudos complementares. Pode indicar, também, a dificuldade, principalmente, dos mestrandos em identificar o limite entre a socialização do conhecimento e registro do conhecimento científico. Vale lembrar que dos 4 doutorandos entrevistados, 3 tiveram como lembrança momentânea títulos de periódicos. Resultado esperado, dada a autonomia do aluno tido como pesquisador, conforme se exige de um doutorando.

Quanto ao fato de títulos periódicos serem mais lembrados é porque possivelmente esses veículos mapeiam o conhecimento do campo e são, portanto, da área de Psicologia.

Do total de 24 títulos de periódicos lembrados, 8 títulos pertencem ao conjunto de periódicos Qualis pesquisados, sendo 7 Qualis A e 1 Qualis B. Dos 8 títulos de periódicos Qualis lembrados, 6 foram citados nas teses/dissertações analisadas.

\section{CONSIDERAÇÕ ES FINAIS}

A análise dos resultados à luz do objetivo principal apontou que a atribuição de conceito máximo

\section{REFERÊNCIAS}

COORDENAÇÃO DE APERFEIÇOAMENTO DE PESSOAL DE NÍVEL SUPERIOR (CAPES). Critérios de avaliação (triênio 19982000). Brasília, 2001. Disponível em: <http://www.capes.gov.br/ portal/conteudo/2000_037_Criterio.pdf > . Acesso em: 28 nov. 2004.

GARVEY, W.D. Communication: The essence of science. Oxford: Pergamon Press, 1979. 332p.

SOUZA, E.P.; PAULA, M.C.S. QUALIS: A base de qualificação dos periódicos científicos utilizada na avaliação da CAPES, a periódicos não induz necessariamente o uso efetivo. O levantamento de citações de periódicos Qualis apontou um uso maior de Qualis B (62,5\%). Tal fato contraria a tendência supostamente almejada de uso de periódicos Qualis A, cujo percentual de uso foi de $53,3 \%$, caso se considere que esses veículos contêm artigos de qualidade e trabalhos de pesquisadores renomados. Conclui-se a partir desse fato que o consenso (avaliação pelos pares) que fundamenta a base e empreendido na classificação de periódicos científicos da área de Psicologia não se reflete no consenso dos segmentos (docentes e discentes) do Programa analisado.

Verifica-se a necessidade de revisão da metodologia utilizada para classificação de periódicos da área de Psicologia, classificação que é usada como instrumento de avaliação da atividade científica dos programas de pós-graduação. A partir desse fato, ficam as recomendações:

- Revisão dos critérios de avaliação e indicadores adotados;

- Maior articulação entre as áreas do conhecimento, para estabelecimento de critérios qualitativos e quantitativos claros e objetivos.
Infocapes, v.10, n.2, p.6-24, 2002. Disponível em: <http:// www.capes.gov.br/capes/portal/conteudo/Infocapes 10_2_2002.pdf>. Acesso em: 8 mar. 2005.

VALÉRIO, P.M. Espelho da ciência: avaliação do Programa Setorial de Publicações em Ciência e Tecnologia da FINEP. Rio de Janeiro/ Brasília: FINEP/IBICT, 1994. 145p.

ZIMAN, J. Conhecimento público. São Paulo: Itatiaia, 1979. $146 p$. 
ANEXO 1

Avaliação de Periódicos Científicos da Área de Psicologia (1998-2000).

\begin{tabular}{|c|c|c|c|}
\hline Periódicos & Conceito & $\begin{array}{c}\text { Escore } \\
\text { Circulação }\end{array}$ & $\begin{array}{c}\text { Escore } \\
\text { Qualidade }\end{array}$ \\
\hline \multicolumn{4}{|l|}{ Nacionais } \\
\hline Psicologia: Reflexão e Crítica & A & 28 & 89 \\
\hline Estudos de Psicologia (UFRN) & A & 28 & 86 \\
\hline Psychê & A & 23 & 80 \\
\hline Arquivos Brasileiros de Psicologia & A & 28 & 77 \\
\hline Interações & A & 19 & 76 \\
\hline Psicologia em Estudo & A & 18 & 76 \\
\hline Psicologia: Teoria e Pesquisa & A & 27 & 74 \\
\hline Rev. Latinoamericana de Psicopatologia Fundamental & A & 23 & 74 \\
\hline Psico (PUCRS) & A & 22 & 73 \\
\hline Estudos de Psicologia (PUC-Campinas) & A & 28 & 68 \\
\hline Estilos da Clínica (USP) & A & 19 & 68 \\
\hline Psicologia: Ciência e Profissão & A & 17 & 68 \\
\hline Paidéia & A & 22 & 63 \\
\hline Psicologia Revista & $A$ & 17 & 63 \\
\hline Psico (USF) & A & 13 & 62 \\
\hline Psicologia USP & B & 13 & 59 \\
\hline Cadernos de Psicologia (UFMG) & B & 18 & 58 \\
\hline Percurso & B & 18 & 57 \\
\hline Psicologia Escolar e Educacional & B & 13 & 54 \\
\hline Aletheia & B & 13 & 54 \\
\hline Temas de Psicologia & B & 13 & 53 \\
\hline Mudanças & B & 18 & 52 \\
\hline Rev. Bras. De Crescimento e Desenvolvimento Humano & B & 13 & 52 \\
\hline Boletim de Psicologia & C & 13 & 49 \\
\hline \multicolumn{4}{|l|}{ Locais } \\
\hline Agora & $A$ & 09 & 58 \\
\hline Psicologia Clínica (PUCRJ) & A & 08 & 49 \\
\hline Cadernos de Psicanálise (SPCRJ) & $A$ & 02 & 46 \\
\hline
\end{tabular}

Fonte: Yamamoto et al. (1999, p.7). Houve a atualização e padronização de siglas. 


\begin{tabular}{|c|c|c|c|}
\hline Periódicos & Conceito & $\begin{array}{c}\text { Escore } \\
\text { Circulação }\end{array}$ & $\begin{array}{c}\text { Escore } \\
\text { Qualidade }\end{array}$ \\
\hline \multicolumn{4}{|l|}{ Locais } \\
\hline Tempo Psicanalítico & $A$ & 4 & 44 \\
\hline Psicologia: Teoria e Prática & $A$ & 1 & 42 \\
\hline Psicologia e Sociedade & $A$ & 9 & 41 \\
\hline Psicologia Argumento & A & 4 & 41 \\
\hline Cadernos de Psicanálise (CPRJ) & B & 2 & 39 \\
\hline Barbarói & B & 12 & 38 \\
\hline Psicanálise e Universidade & B & 9 & 35 \\
\hline Scientia Sexualis & B & 5 & 33 \\
\hline Opção Lacaniana & B & 4 & 33 \\
\hline Interação & B & 8 & 31 \\
\hline Ciência Cognitiva & B & 7 & 31 \\
\hline Psicologia da Educação & B & 4 & 30 \\
\hline Mente Social & B & 1 & 30 \\
\hline Psicólogo Informação & B & 0 & 30 \\
\hline Cadernos do Tempo Psicanalítico & C & 6 & 28 \\
\hline Cadernos de Psicologia SBP & C & 6 & 27 \\
\hline Revista do Dept. de Psicologia da UFF & C & 0 & 27 \\
\hline Cadernos de Psicologia (UERJ) & C & 0 & 27 \\
\hline Série Documenta & C & 1 & 23 \\
\hline Perfil & C & 2 & 21 \\
\hline Interfaces & C & 11 & 17 \\
\hline Vertentes & C & 0 & 14 \\
\hline Psicanálise e Debate & C & 6 & 10 \\
\hline
\end{tabular}

Tempo Psicanalítico

Psicologia: Teoria e Prática

Psicologia e Sociedade

de Psicanálise (CPRJ)

Barbarói

Psicanálise e Universidade

Fonte: Yamamoto et al. $(1999$, p.7). Houve a atualização e padronização de siglas. 
